\title{
Modification of Gene Expression of Connexins in the Rat Corpus Epididymis by Estradiol Benzoate or Flutamide Exposure at the Early Neonatal Age
}

\author{
${ }^{\dagger}$ Ki-Ho Lee \\ Dept. of Biochemistry and Molecular Biology, College of Medicine, Eulji University, Daejeon 301-746, Korea
}

\begin{abstract}
Cell-cell direct communication through channel-forming molecules, connexin $(C x)$, is essential for a tissue to exchange signaling molecules between neighboring cells and establish unique functional characteristics during postnatal development. The corpus epididymis is a well-known androgen-responsive tissue and involves in proper sperm maturation. In the present research, it was attempted to determine if expression of $C x$ isoforms in the corpus epididymis in the adult is modulated by exposure to estrogenic or anti-androgenic compound during the early postnatal period. The neonatal male rats at 7 days of age were subcutaneously injected by estradiol benzoate (EB) at low-dose $(0.015 \mu \mathrm{g} / \mathrm{kg}$ body weight $)$ or high-dose $(1.5 \mu \mathrm{g} / \mathrm{kg}$ body weight) or flutamide (Flu) at low-dose $(500 \mu \mathrm{g} / \mathrm{kg}$ body weight) or high-dose $(50 \mathrm{mg} / \mathrm{kg}$ body weight). The corpus epididymis collected at 4 months of age was subjected to evaluate expressional changes of $C x$ isoforms by quantitative real-time PCR. Treatment of low-dose EB resulted in increases of $C x 32, C x 37$, and $C x 45$ transcript levels, while exposure to high-dose EB decreased expression of $C x 26, C x 30.3, C x 31, C x 31.1, C x 32, C x 40, C x 43$, and $C x 45$. Treatments of Flu caused significant decreases of expression of all examined $C x$ isoforms, except $C x 37$ and $C x 43$ shown no expressional change with high-dose Flu treatment. These findings imply that expression of most $C x$ isoforms present in the corpus epididymis would be transcriptionally regulated by actions of androgen and/or estrogen during postnatal period.
\end{abstract}

Key words : Connexin, Estradiol benzoate, Flutamide, Gene expression, Corpus epididymis

\section{INTRODUCTION}

The epididymis plays important roles in male reproduction, including sperm maturation, transport, and storage (Robaire \& Hermo, 1988). The epididymis has a tubular structure covered by an epithelial layer, consisting of several cell types (Arrotéia et al., 2012). Except the initial segment at the most proximal region of the epididymal region, the epididymis is divided into three parts, caput (head), corpus (body), and cauda (tail) epididymis, depending on functional, morphological, and molecular biological characteristics (Robaire \& Hermo, 1988). The most predominant type of the epithelial cells in the epididymis is principal cells which are columnar shape with stereocilia protruding into the lumen (Robaire \& Hermo, 1988). The number of principal cells gradually decreases from the caput to the cauda epididymis (Robaire \& Hermo, 1988). However, the epithelial content of narrow cells increases from the caput to the corpus epididymis, while clear cells are relatively equally distributed through the entire epididymis (Arrotéia et al., 2012). It is generally considered that differrent cell types in the epididymis play specific functions on

\footnotetext{
Manuscript received 3 April 2015, Received in revised form 20 April 2015, Accepted 20 May 2015

${ }^{\dagger}$ Corresponding Author : Ki-Ho Lee, Dept. of Biochemistry and Molecular Biology and Medical Sciences Research Institute, Eulji University, Daejeon, 301-746, Korea. Tel. : +82-42-259-1643, Fax : +82-42-259-1649, E-mail : kiholee@eulji.ac.kr

This is an Open Access article distributed under the terms of the Creative Commons Attribution Non-Commercial License (http:// creativecommons.org/licenses/by-nc/3.0) which permits unrestricted non-commercial use, distribution, and reproduction in any medium, provided the original work is properly cited.
} 
the establishment of unique epididymal microenvironment (Robaire et al., 2006). Thus, it is reasonable to consider that the different ratios of cell population in the epididymal segment would partly contribute into creation of discrete functional epididymal unit.

Exchanges of signaling molecules, ions, and even RNAs through connexin $(C x)$-based gap junction between neighboring cells is a type of direct cell-cell communication ways (Goodenough et al., 1996). There are at least $20 C x$ isoforms found in mammals (Meșe et al., 2007). The gap junction is composed of a complex of two hemichannels, which each is made of six $C x$ isoforms (Cyr, 2011). The presence of $C x$ isoforms in the male reproductive tract has been examined by several researchers, including our recent findings. Expression of $C x$ transcripts in the reproductive excurrent duct has been detected in the initial segment (Seo et al., 2010) and epididymis (Han \& Lee, 2013; Lee, 2013). Even though expressional pattern of each $C x$ is quite distinguishable from each other isoform during postnatal development, there are nine $C x$ isoforms expressing throughout the entire epididymis, including $C x 26, C x 30.3, C x 31$, Cx31.1, Cx32, Cx37, Cx40, Cx43, and Cx45 (Han \& Lee, 2013; Lee, 2013). Expression of certain $C x$ isoform in the epididymis shows the segment-specific patterns (Han \& Lee, 2013; Lee, 2013). For example, the highest levels of Cx40 are detected at 45 days of age in the caput epididymis, at early than 45 days of age in the corpus epididymis, and 5 months of age in the cauda epididymis (Han \& Lee, 2013; Lee, 2013). It is difficult to generalize expressional patterns of $C x$ isoforms in the epididymis during postnatal period because expression of each $C x$ isoform shows a unique pattern in each epididymal segment. Each $C x$ isoform possesses particular permeability and gating behavior to specific signaling molecules (Bruzzone et al., 1996). Therefore, it is logic to consider that expression of various $C x$ isoforms at different levels in the epididymal segments would be necessary to communicate different cell types and thus to produce distinct microenvironment within the epididymal part. Even though a few researches show immuno-localization of some $C x$ isoforms at specific cell types within the epididymis (Cry, 2011), the function of $C x$ isoforms has not been clearly demonstrated.

Expressional regulation of $C x$ isoforms in the epididymis has not been extensively examined, in spite of the presence of strong evidence demonstrating expressional modulation of a number of genes in the epididymis by estrogens and androgens. Dubé et al (2012) have showed possible regulation of $C x 43$ expression in the human cauda epididymis by epidermal growth factor. Our previous researches have demonstrated that exposure to estradiol benzoate (EB) or flutamide (Flu) at the early or weaning postnatal age results in altered expression of several $C x$ isoforms in the initial segment of the adult rat epididymis (Lee, 2014; 2015). However, scientific research showing hormonal factor(s) giving an influence on $C x$ expression in the corpus epididymis is very rare. Thus, in accordance with our earlier studies (Lee, 2014; 2015), the present research was attempted to reveal the effect of $\mathrm{EB}$, an estrogenic agonist, or Flu, an antiandrogenic agent, on expression of $C x$ isoforms in the corpus epididymis. Expressional changes of $C x$ isoforms in the adult corpus epididymis followed by the subcutaneous expose to these steroidal compounds at 7 days of postnatal age were evaluated by quantitative realtime PCR analyses.

\section{MATERIALS AND METHODS}

1. Preparation and treatment of estradiol benzoate (EB) and flutamide (Flu)

The EB and Flu were purchased from Tokyo Chemical Industry Co. (Tokyo, Japan). The powder of EB or Flu was dissolved in $100 \% \mathrm{EtOH}$ at room temperature. The final stock solutions of EB and Flu were prepared with a series 
of dilution in peanut oil.

A total of five pregnant Spragure Dawley rats were obtained from Samtako (OSan, Korea). Each female rat was individually kept during entire experimental period. Free access to drinking water and food was allowed. Each rat was randomly assigned to one of five experimental groups, including control (peanut oil), low-dose EB treated (EB-L), high-dose EB treated (EB-H), low-dose Flu treated (Flu-L), or high-dose Flu treated (Flu-H) group. Each pregnant rat delivered 5 to 8 male pups, which were treated with EB or Flu at 7 days of postnatal age. Subcutaneous injection of EB or Flu was carried out at the final concentration of $0.015 \mu \mathrm{g}(\mathrm{EB}-\mathrm{L})$ or $1.5 \mu \mathrm{g}(\mathrm{EB}-\mathrm{H})$ per kilogram of body weight (/kg BW) for EB or $500 \mu \mathrm{g}$ (Flu-L) or $50 \mathrm{mg}(\mathrm{Flu}-\mathrm{H}) / \mathrm{kg} \mathrm{BW}$ for Flu. Number of animals for each experiment group are as following; control $(n=5)$, EB-L $(n=7)$, EB-H $(n=8)$, Flu-L $(n=7)$, and Flu-H $(n=7)$. The present study was carried out in accordance with the guide for the care and use of laboratory animals of National Research Council in S. Korea.

\section{Total RNA and cDNA preparation}

The epididymis was collected from the experimental animals at 4 months of age. First, the animals were anesthetized by $\mathrm{CO}_{2}$ stunning in a closed chamber. The testis and epididymis were exposed through a lower abdominal incision. In cold PBS, the epididymis was separated from the testis and further dissected into initial segment, caput epididymis, corpus epididymis, and cauda epididymis. The epididymal parts were washed in fresh cold PBS for a couple of times and frozen in liquid nitrogen.

Total RNA from the corpus epididymis was harvested with $0.5 \mathrm{~mL}$ of easy-Blue total RNA extraction solution (iNtRON Biotechm Seongnam, Korea). Tissue was first homogenized in the solution with a polytron homogenizer (Fisher Scientific, Pittsburgh, USA). After then, phenolchloroform extraction protocol was applied to obtain a pellet of total RNA. Total RNA was resuspended in RNasefree $\mathrm{dH}_{2} \mathrm{O}$, and purity and concentration of total RNA were determined spectrophotometrically (Eppendorf, New York, USA).

Reverse transcription (RT) reactions to generate the first strand of cDNAs were carried out with oligo-dT primer and ImProm-II ${ }^{\mathrm{TM}}$ reverse transcription system (Promega, Madison, USA). One microgram of total RNA was utilized as a template, and RT reactions were performed at $25^{\circ} \mathrm{C}$ for $5 \mathrm{~min}, 42^{\circ} \mathrm{C}$ for $1 \mathrm{hr}$ and $30 \mathrm{~min}$, and $70^{\circ} \mathrm{C}$ for $15 \mathrm{~min}$.

\section{Quantitative real-time PCR}

One microliter of $20 \mu \mathrm{L}$ RT reaction mixtures was used to carry out real-time PCR. The PCR cocktail included 10 pmol of primer set, $10 \mu \mathrm{L}$ of master mixture (Finnzymes, Espoo, Finland), and DNase-free water to make a final volume of $20 \mu \mathrm{L}$. The $\mathrm{CFX} 96^{\mathrm{TM}}$ real-time PCR detection system (Bio-rad, Hercules, USA) was used for real-time PCR analysis. The specificity of each PCR was determined by the melting curve analysis. The following primers were used for quantitative real-time PCR. Cx30.3 (NM_053984) primers: forward: CCCAATGTCTGCTATGACGA; reverse: CACAGCAGCCTTGAAGATGA, Cx31 (NM_019240) primers: forward: TTGAGCGGTGTGAACCAGTA; reverse: TGTTGGAGATGGGGAAGAAG, Cx31.1 (NM_019241) primers: forward: CATCGTCTGCATCCTGCTTA; reverse: ATGAGGTCGCTTGAGAGGAA, Cx32 (NM_017251) primers: forward: AGAATCATGGTGCTGGTGGT; reverse: CCTCAAGCCGTAGCATTTTC, Cx37 (NM_021654) primers: forward: AGTGTCTGTACCTTGGATGCC; reverse: CAGCACACTTAGCCAAGAGC, Cx40 (NM_019280) primers: forward: ATACCATTCAGCCTGGTTGC; reverse: CGGCCTCTTTAGCTTTCTCA, Cx43 (NM_012567) primers: forward: AGCAAGCTAGCGAGCAAAAC; reverse: GAG TTCATGTCCAGCAGCAA, CX45 (NM_001085381) primers: forward: GATCATCCTGGTTGCTACTC; reverse: GATC CTCTTCATGGTCCTCT, and cyclophilin A (Ppia) primers: 
forward: GGCAAATGCTGGACCAAACAC; reverse: TT AGAGTTGTCCACAGTCGGAGATG. The PCR was performed at a cycle of $95^{\circ} \mathrm{C}$ for $30 \mathrm{sec}$ and cycles of denaturation at $95^{\circ} \mathrm{C}$ for $30 \mathrm{sec}$, annealing at melting temperature for $30 \mathrm{sec}$, and extension at $72^{\circ} \mathrm{C}$ for $30 \mathrm{sec}$. Each PCR was completed with an additional extension at $72^{\circ} \mathrm{C}$ for $30 \mathrm{sec}$.

\section{Statistical analysis}

Triplicated PCRs were performed for each cDNA, and three to four cDNAs were used for each experimental group. Following densitometric analysis, signal intensities of $C x$ isoforms were normalized to internal controls (Ppia). One-way ANOVA was carried out to check significant differences among experimental groups, and Duncan's test, a post-hoc analysis, was performed to determine statistical significances between control and EB-treat or Flu-treated groups. When $P$ value was lower than 0.05 , it was considered to be significant.

\section{RESULTS}

1. Expressional modification of $C \times 26$ and $C \times 30.3$ in the corpus epididymis exposed to EB or Flu at 7 days of postnatal age

Expression of $C \times 26$ in the adult corpus epididymis was not changed by treatment of EB at low dose (Fig. 1A). However, compared with that of control, the treatment of high-dose EB resulted in a significant decrease of Cx26 transcript level in the adult corpus epididymis (Fig. 1A). Interestingly, regardless of dose of administration, the treatments of Flu at 7 days of age caused significant decreases of $C x 26$ expression in the adult corpus epididymis (Fig. 1A). Similar findings were observed in expression patterns of $C x 30.3$ (Fig. 1B). Even though there was no expressional change of $C \times 30.3$ by low-dose EB treatment at 7 days of age, a significant decrease of $C \times 30.3$ transcript
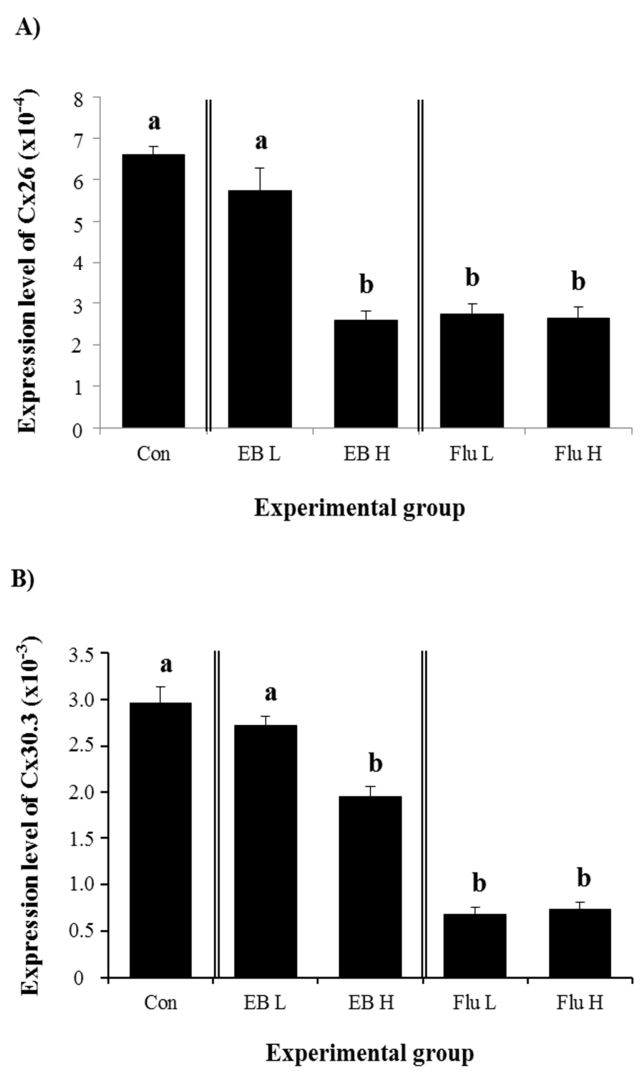

Fig. 1. Expression patterns of $C x 26$ and $C x 30.3$ in the corpus epididymis of adult rat. Seven-days old of male rats were treated with either estradiol benzoate at low dose (EB L, $0.015 \mu \mathrm{g} / \mathrm{kg} \mathrm{BW}$ ) or high dose (EB H, $1.5 \mu \mathrm{g} / \mathrm{kg} \mathrm{BW}$ ) or flutamide at low dose (Flu L, $500 \mu \mathrm{g} / \mathrm{kg} \mathrm{BW}$ ) or high (Flu H, $50 \mathrm{mg} / \mathrm{kg}$ BW) dose. Statistical differences among experimental groups on transcript levels of Cx26 (A) or Cx30.3 (B) at $p<0.05$ level are shown in different letters

level in the adult corpus epididymis was detected by highdose EB treatment at 7 days of postnatal age (Fig. 1B). Treatment of Flu at low- or high-dose at seven-day of old resulted in significant decrease of $C \times 30.3$ expression in the adult corpus epididymis (Fig. 1B).

2. Expressional modification of $C \times 31$ and $C \times 31.1$ in the corpus epididymis exposed to EB or Flu at 7 days of postnatal age

Treatment of low-dose of EB at 7 days of age didn't 
A)

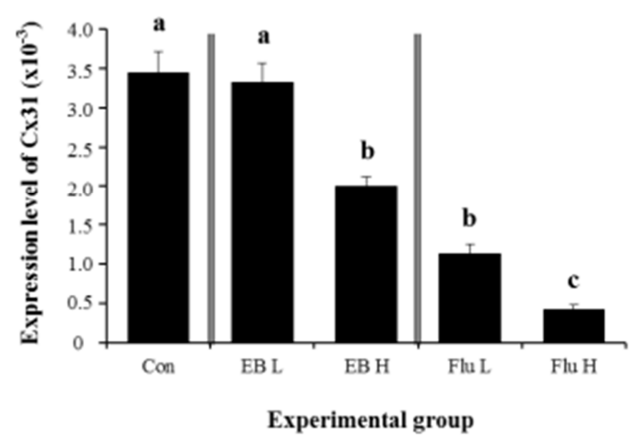

B)

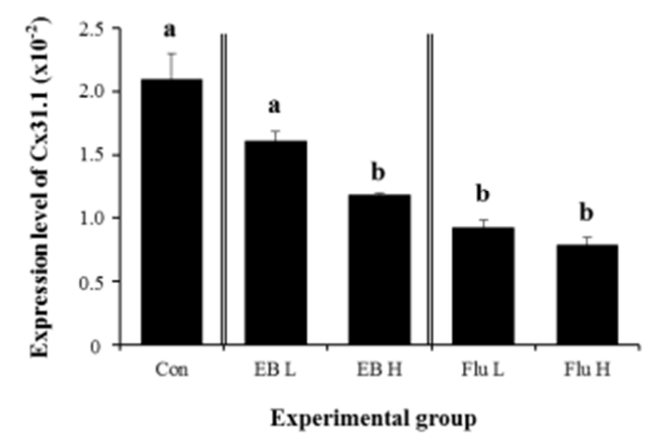

Fig. 2. Expression patterns of $C x 31$ and $C x 31.1$ in the corpus epididymis of adult rat. Seven-days old of male rats were treated with either estradiol benzoate at low dose (EB L, $0.015 \mu \mathrm{g} / \mathrm{kg} \mathrm{BW}$ ) or high dose (EB H, $1.5 \mu \mathrm{g} / \mathrm{kg} \mathrm{BW}$ ) or flutamide at low dose (Flu L, $500 \mu \mathrm{g} / \mathrm{kg} \mathrm{BW}$ ) or high (Flu H, $50 \mathrm{mg} / \mathrm{kg}$ BW) dose. Statistical differences among experimental groups on transcript levels of $C x 31$ (A) or $C x 31.1$ (B) at $p<0.05$ level are shown in different letters

give an influence on transcription of $C x 31$ in the corpus epididymis at the adult (Fig. 2A). However, a decrease of Cx31 transcript level was detected by high-dose EB treatment in the corpus epididymis (Fig. 2A). Exposure to lowdose Flu at 7-days old of age resulted in a significant decrease of $C x 31$ expression in the corpus epididymis (Fig. 2A). A further decrease of $C x 31$ expression was found in a treatment of high-dose Flu (Fig. 2A). Administration of EB at a low-dose at 7 days of age didn't cause aberrant expression of $C x 31.1$ in the adult corpus epididymis, even though treatment of high-dose EB led to a significant de- crease of $C x 31.1$ expression (Fig. 2B). Treatments of Flu at two doses resulted in significant decreases of $C x 31.1$ transcript levels, compared with that of control group (Fig. 2B)

\section{Expressional modification of $C \times 32$ and $C \times 37$ in} the corpus epididymis exposed to EB or Flu at 7 days of postnatal age

A significant increase of $C x 32$ expression was found in the corpus epididymis treated with low-dose of EB at 7 days of age (Fig. 3A). However, treatment of high-dose

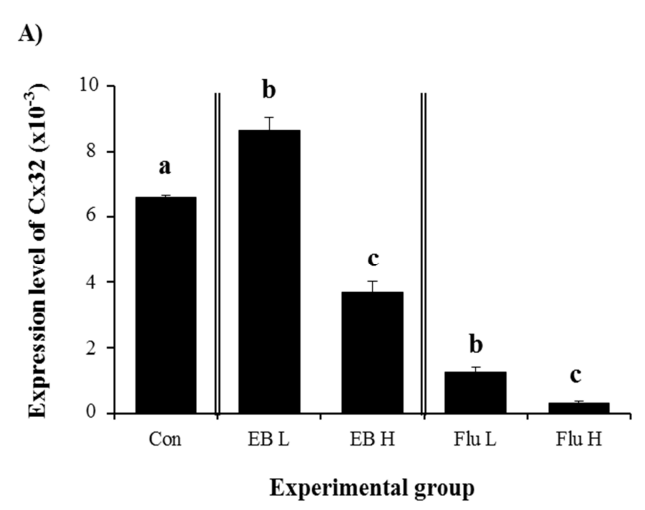

B)

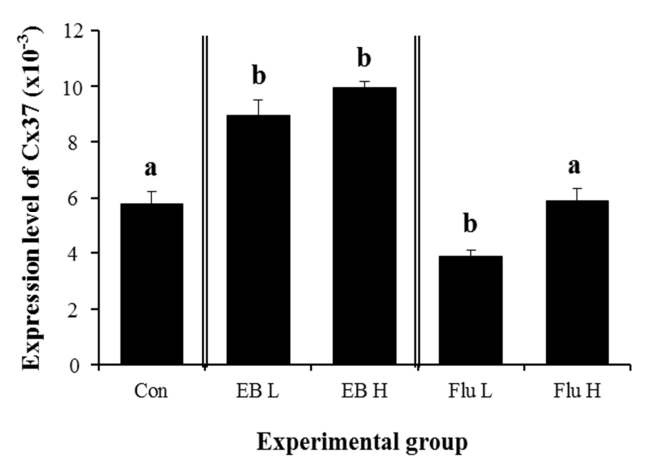

Fig. 3. Expression patterns of $C x 32$ and $C x 37$ in the corpus epididymis of adult rat. Seven-days old of male rats were treated with either estradiol benzoate at low dose (EB L, $0.015 \mu \mathrm{g} / \mathrm{kg} \mathrm{BW}$ ) or high dose (EB H, $1.5 \mu \mathrm{g} / \mathrm{kg} \mathrm{BW}$ ) or flutamide at low dose (Flu L, $500 \mu \mathrm{g} / \mathrm{kg} \mathrm{BW}$ ) or high (Flu H, $50 \mathrm{mg} / \mathrm{kg}$ BW) dose. Statistical differences among experimental groups on transcript levels of $C x 32$ (A) or $C x 37$ (B) at $p<0.05$ level are shown in different letters 
EB caused a transient decrease of $C x 32$ transcript level in the adult corpus epididymis (Fig. 3A). A great decrease of Cx32 expression was detected with low-dose Flu treatment (Fig. 3A). Expression of $C x 32$ in the adult corpus epididymis was barely detected with a treatment of high-dose Flu (Fig. 3A). Interestingly, treatments of EB at 7 days of age caused expressional increases of $C x 37$ in the adult corpus epididymis (Fig. 3B). However, an administration of low-dose Flu resulted in a significant decrease of $C x 37$ expression, while there was no change of Cx37 transcript level with high-dose Flu treatment (Fig. 3B).

\section{Expressional modification of $C x 40, C x 43$, and} Cx45 in the corpus epididymis exposed to EB or Flu at 7 days of postnatal age

Expressional change of $C x 40$ in the adult corpus epididymis after exposure to EB at 7 days of age was only detected with high-dose EB treatment (Fig. 4A). Treatment of Flu at low- or high-dose resulted in significant decrease of Cx40 transcript level, compared with that of control (Fig. 4A). A significant decrease of $C x 43$ transcript level was observed with high-dose EB treatment, while no change of Cx43 expression was found in a treatment of low-dose EB treatment (Fig. 4B). Treatment of low-dose Flu at 7 days of age resulted in a significant decrease of $C x 43$ expression in the adult corpus epididymis (Fig. 4B). But, expression of $C x 43$ in the corpus epididymis was not influenced by high-dose Flu treatment (Fig. 4B). Expression of Cx45 in the adult corpus epididymis was significantly increased by low-dose EB treatment but was significantly decreased by high-dose EB treatment (Fig. 4C). Even though Flu treatments caused significant decreases of $C x 45$ expression in the corpus epididymis, a further significant decrease of Cx45 transcript level was detected with high-dose treatment of Flu (Fig. 4C).

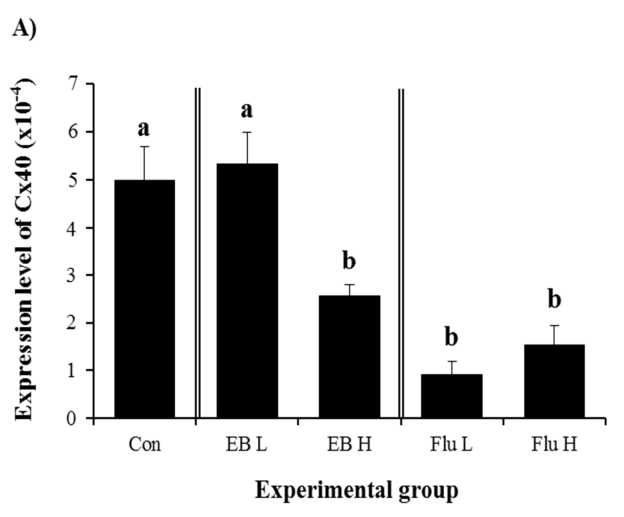

B)
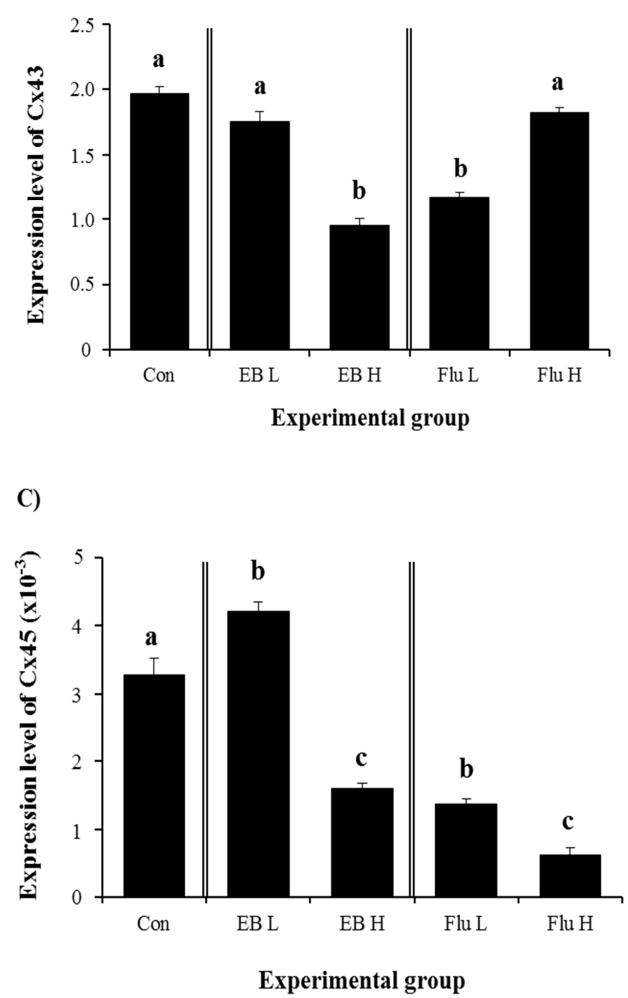

Fig. 4. Expression patterns of $C x 40, C x 43$, and $C x 45$ in the corpus epididymis of adult rat. Seven-days old of male rats were treated with either estradiol benzoate at low dose (EB L, $0.015 \mu \mathrm{g} / \mathrm{kg} \mathrm{BW}$ ) or high dose $(\mathrm{EB} \mathrm{H}, 1.5 \mu \mathrm{g} / \mathrm{kg} \mathrm{BW})$ or flutamide at low dose (Flu L, $500 \mu \mathrm{g} / \mathrm{kg} \mathrm{BW}$ ) or high (Flu H, 50 $\mathrm{mg} / \mathrm{kg} \mathrm{BW}$ ) dose. Statistical differences among experimental groups on transcript levels of $C x 40$ (A), $C x 43$ (B), or Cx45 (C) at $p<0.05$ level are shown in different letters 


\section{DISCUSSION}

Disruption of hormonal homeostasis by exogenous administration of EB or Flu to male commonly develops abnormal expression of a number of genes related with functions of the epididymis (Gorowska et al., 2014; Kaushik et al., 2010). In addition, neonatal exposure of steroidal compounds often results in permanent changes of male reproductive function at the adult (Goyal et al., 2003). The present study was designed to determine the effect of EB or Flu treatment at neonatal age on expression of $C x$ isoforms in the corpus epididymis of adult rats.

It is likely that treatment of low-dose EB at neonatal age doesn't greatly affect expression of most $C x$ isoforms in adult corpus epididymis, except $C x 32, C x 37$, and $C x 45$, which were significantly increased. These findings were somewhat different from those observed in the initial segment (IS), which treatment of low-dose EB caused significant increases of $C x 30.3, C x 31.1$, and $C x 43$ expression but no other $C x$ isoforms (Lee, 2014). These results suggest that expression of $C x$ isoform in the IS and corpus epididymis is differentially regulated by low-dose EB. The level of estrogen receptor (ER) $\alpha$ along the epididymis varies with the highest at the corpus than other epididymal regions (Hess et al., 2011). Such differential levels of ER $\alpha$ among the epididymal segments would contribute to differrent expressional patterns of $C x$ isoforms induced by lowdose EB treatment. It is also possible that responsiveness to low-dose $\mathrm{EB}$ on expression of $C x$ isoform would be different among epididymal regions. At this moment, the current findings couldn't provide firm evidence to answer such differential expression of $C x$ isoforms by low-dose EB treatment between the IS and corpus epididymis. Molecular dissection of promoter regions of $C x$ isoforms would offer a possibility to understand how such differential responsiveness on expression of $C x$ isoforms to low-dose EB occurs within different epididymal regions.
Exposure to high-dose EB at the neonatal age generally led to significant decreases of $C x$ isoforms in adult corpus epididymis, except $C x 37$ with a significant increase. These results relatively differ from those detected in the IS, which expression of $C x$ isoform in the adult after neonatal high-dose EB treatment didn't show any specific pattern (Lee, 2014). Aberrant expression of $C x$ isoform by highdose EB would be partly explained by differential responsiveness of $C x$ genes at the dose of EB. From the findings from current study, it is generally considered that treatment of $\mathrm{EB}$ at $1.5 \mu \mathrm{g} / \mathrm{kg} \mathrm{BW}$ at 7 days of age is sufficient to suppress expression of most $C x$ isoforms in adult corpus epididymis, even though an exception is $C x 37$. One might think that the treatment of high-dose EB at neonatal age has a disruptive effect on general hormonal balance and thus causes expressional decreases of most $C x$ isoforms. But, because expression pattern of $C \times 37$ is not the case, it is more thoroughly considered that expressional regulation of $\mathrm{Cx} 37$ by $\mathrm{EB}$ would differ from other $C x$ isoforms in the corpus epididymis.

Administration of low-dose Flu at 7 days of age resulted in significant expressional decreases of all $C x$ isoforms in adult corpus epididymis. Even though the same treatment of Flu results in similar results in the IS, expression of some $C x$ isoforms in the IS are not changed or significantly increased (Lee, 2014). These conflict results observed between the IS and corpus epididymis would be fairly explained by segment-specific expressional regulation of some $C x$ isoforms by low-dose Flu. The level of androgen receptor $(A R)$ in the corpus epididymis is the lowest among epididymal segments (Pujol \& Bayard, 1979). As an antiandrogenic compound, the Flu acts on suppression of AR signaling pathway. Thus, it is reasonable to think that expression of $C x$ isoforms in the corpus epididymis is up-regulated by androgen. And, disruption of hormonal homeostasis by exogenous exposure of an antiandrogen at the neonatal age could result in permanent defect on 
expression of $C x$ isoforms in the adult. Additional researches are suggested to resolve androgenic regulation on expression of $C x$ isoforms in the corpus epididymis.

Expression of $C x$ isoforms in adult corpus epididymis after high-dose Flu treatment at neonatal age is somewhat unusual. As might expected, expression of most $C x$ isoforms is significantly lower than the control or further decreased than that with low-dose Flu treatment. However, the levels of $C \times 37$ and $C x 43$ do not differ from those of control. These observations are surprisingly and are not simply answered with the findings obtained from present research. A possible suggestion would be addressed from negative dose-dependent expressional regulation of genes. Also, it is considerable that high-dose Flu could influence different signaling pathway at which low-dose Flu does not give an impact. But, it is clear that expressional regulation of $C \times 37$ and $C \times 43$ by androgen is more complicate than expected. More detailed examination is required for providing defined data on $C x 37$ and $C x 43$ expression in the corpus epididymis.

In conclusion, the present research has clearly demonstrated that neonatal exposure to $\mathrm{EB}$ or Flu results in aberrant expression of $C x$ isoforms in adult corpus epididymis. The epididymis is a major site for sperm maturation, and $C x$ is particularly important for communicating between different cell types, such as the epididymis. Even though it is not considered at the present research, additional investigations are suggested to examine the effect of EB or Flu treatment at neonatal age on male fertility.

\section{ACKNOWLEDGEMENT}

This research was supported by Basic Science Research Program through the National Research Foundation of Korea (NRF) funded by the Ministry of Education, Science and Technology (20090067187 and 20100022388).

\section{REFERENCES}

Arrotéia KF, Garcia PV, Barbieri MF, Justino ML, Pereira LAV (2012) The epididymis: embryology, structure, function and its role in fertilization and infertility. In: Pereira LAV (ed). Embryology-Updates and Highlights on Classic Topics. In Tech, Croatia, pp 41-66.

Bruzzone R, White TW, Paul DL (1996) Connections with connexins: the molecular basis of direct intercellular signaling. Eur J Biochem 238:1-27.

Cyr DG (2011) Connexins and pannexins: coordinating cellular communication in the testis and epididymis Spermatogenesis 1:325-338.

Goodenough DA, Goliger JA, Paul DL (1996) Connexins, connexons, and intercellular communication. Annu Rev Biochem 65:475-502

Gorowska E, Zarzycka M, Chojnacka K, Bilinska B, Hejmej A (2014) Postnatal exposure to flutamide affects CDH1 and CTNNB1 gene expression in adult pig epididymis and prostate and alters metabolism of testosterone. Andrology 2:186-197.

Goyal HO, Robateau A, Braden TD, Williams CS, Srivastava KK, Ali K (2003). Neonatal estrogen exposure of male rats alters reproductive functions at adulthood. Biol Reprod 68:2081-2091

Han SY, Lee K-H (2013) The expression patterns of connexin isoforms in the rat caput epididymis during postnatal development. J Ani Sci Tech 55:245-255

Hess RA, Fernandes SA, Gomes GR, Oliveira CA, Lazari MF, Porto CS (2011) Estrogen and its receptors in efferent ductules and epididymis. J Androl 32:600-613.

Kaushik MC, Misro MM, Sehgal N, Nandan D (2010) Effect of chronic oestrogen administration on androgen receptor expression in reproductive organs and pituitary of adult male rat. Andrologia 42:193-205.

Lee K-H (2013) Differential expression of multiple connexins in rat corpus and cauda epididymis at various postnatal stages. J Ani Sci Tech 55:521-530. 
Lee K-H (2014) Expressional modulation of connexin isoforms in the initial segment of male rat treated with estradiol benzoate or flutamide. Balsaenggwa Saengsig 18:293300

Lee K-H (2015) Exogenous exposure to estradiol benzoate or flutamide at the weaning age alters expression of connexin isoforms in the initial segment of male rat. Balsaenggwa Saengsig 19:43-51.

Meșe G. Richard G, White TW (2007) Gap junctions: basic structure and function. J Invest Dermatol 127:2516-2524.

Pujol A, Bayard F (1979) Androgen receptors in the rat epididymis and their hormonal control. J Reprod Fert 56:217-222.
Robaire B, Hermo L (1988) Efferent ducts, epididymis, and vas deferens: structure, functions, and their regulation. In: Knobil E et al (eds.). The Physiology of Reproduction. Raven Press, New York, NY, pp 999-1080.

Robaire B, Hinton BT, Orgebin-Crist MC (2006) The epididymis. In: Knobil E and Neil J (eds.). The Physiology of Reproduction, Elsevier, New York, NY pp 10711148.

Seo H-H, Seon C-W, Choi I, Cheon Y-P, Cheon T-H, Lee $\mathrm{K}-\mathrm{H}$ (2010) Expressional profiling of connexin isoforms in the initial segment of the male reproductive tract during postnatal development. Reprod Dev Biol 34: 103-109. 